\title{
Surgical randomized controlled trials: reflection of the difficulties
}

\author{
Lydia Masako Ferreira
}

Randomized controlled trials (RCTs) have been used in medical research to evaluate different interventions in health-care. It has been shown that human clinical trials that lack randomization (RND) or blinding (BLD) often overestimate the magnitude of treatment effects ${ }^{1}$.

There are concerns that the use of the term "randomized" conveys a form of legitimacy to surgical trials that may sometimes be inappropriate and some published surgical trials ignore basic aspects of the randomization process. In this area, there are many reasons for it, as it is difficult for surgeons to have faith in trials that fail to demonstrate an unbiased allocation of patients and ignore the need to maintain some confidentiality about the allocation of patients into groups.

Researchers usually randomize an equal number of subjects to each study group by random allocation and restricted randomization (permuted block design). In random allocation, numbers can be randomly generated by a computer or selected in sequence from a table of random numbers. Although this method of randomization results in approximately equal sample sizes per group, there is a slight possibility of a very unequal distribution among the groups. Some discrepancy between the numbers in the comparison groups would be expected. This possibility is increased with small sample sizes. If this occurs, the investigator may want to generate an entirely new randomization list. This is known as replacement randomization, and is preferable to selectively replacing individual treatment assignments (that ruins the randomness of the list). An alternative is to use a permuted block design. A permuted block design is used to ensure an equal number of subjects for each treatment group at specified intervals throughout the study. Subjects are assigned in blocks of a specified number, with an equal number of subjects in each treatment group within each block. This method is used to avoid treatment group imbalances in size if the study is stopped prematurely, before the total predetermined sample size is reached ${ }^{1}$.

Forcing equal group sizes potentially harms the unpredictability of treatment assignments, especially when using permuted-block randomization in non-double-blinded trials. Investigators underuse simple randomization and overuse fixed-block randomization ${ }^{2}$.

In simple, unrestricted, randomized trials, the sizes of groups should indicate random variation. The appeal of equal group sizes in a simple randomized controlled trial is cosmetic, non-scientific. There are plenty of excellent textbooks on how to conduct RCTs the right way ${ }^{4-8}$. They intend to help clinicians advise the patient who is eligible for, or has been invited to join, an RCT, by showing both of them how to determine whether the proposed trial is likely to generate results that are valid, clinically useful and of sufficient value to the patients for them to consider joining it. These essays intend to help clinicians and patients decide whether the report of an RCT is true enough and clinically sensible enough for its results to be offered by clinicians and requested by patients. And the objective is to help clinicians at any stage of their training in an RCT and want to sidestep the avoidable errors.

In spite of the RCT's usefulness in promoting health, limiting harm and playing a dominant role in medical researches, why is it that many surgeons cannot randomize surgical treatments adequately? Answers to this question may include many issues related to surgery itself and patients ${ }^{3,9}$. Reasons for the randomization constraints found in surgical trials include: 1 . variations in an operation may occur during the operation itself and these variations can often influence the outcome; 2 . difficulty to assess the technical quality of an operation; 3. outcomes may be more strongly related to the skills of a particular surgeon rather than the technique used; 4 . some surgical techniques are rare and the surgeon cannot accumulate sufficient sample sizes; 5 . the learning curve for new techniques make it impractical for two different surgical methods to be employed simultaneously; 6 . the trial should be continued well beyond 
the end of the learning curve; 7. each surgeon's different experiences; 8. many surgical trials cannot be double blinded; 9. the use of placebos in randomized surgical trials is extremely controversial; 10. outcomes may require years of follow-up; 11. emergency surgery often makes consent and randomization is very difficult; 12 . practical difficulties in following procedures; 13 . there are no significantly government grants or commercial sponsors for surgical research ${ }^{10,11}$.

Patient-related issues also contribute to the obstacles found in randomizations in surgical trials: 1 . patients may refuse to be randomized; 2 . patients may be unwilling to allow randomization to choose the type of surgery ${ }^{9} ; 3$. doctor-patient relationship would be affected by a randomized clinical trial; 4 . difficulty with informed consent; 5. dislike of open discussions involving uncertainty; 6. perceived conflict between the roles of scientist and clinician; 7. feelings of personal responsibility if the treatments were found to be unequal; 8. difficulty in recruiting patients, particularly in poor countries.

These constraints explain why surgical trials are complex and, sometimes impossible to randomize. To obtain meaningful results in any clinical trial, patients need to be allocated to treatments in such a way that valid analysis can be carried out. Balancing treatment groups before data analysis is more desirable than trying to compensate for incomparability at a later date. Therefore, the development of allocation procedures to produce comparable groups in which prognostic factors are equally represented is important. Minimization, a deterministic allocation method, aims to ensure balance on such factors, particularly in small trials when traditional randomization methods are likely to fail ${ }^{12,13}$. The proposed strategies should focus on how RCTs should be designed and executed, and they should challenge both the assumptions and the behavior of contemporary trialists, ethics committees and trial monitors.

\section{References}

1. Bebarta V, Luyten D, Heard K - Emergency medicine animal research: does use of randomization and blinding affect the results? Acad Emerg Med. 2003;10(6):684-7.

2. Garb JL - Understanding Medical Research. A prationer's guide. Boston: Little, Brown and Company Inc; 1996.

3. Russel I - Evaluating new surgical procedures. BMJ. 1995;311(7015):1243-4.

4. Hailey D - Scientific harassment by pharmaceutical companies: time to stop. CMAJ. 2000;162(2):2123. Available: www.cma.ca/cmaj/vol-162/issue-2/0212.htm[Full Text]

5. Schwartz D, Flamant R, Lellouch J - Clinical trials [translated by Healy MJR]. Toronto: Academic Press; 1980.

6. Friedman LM, Furberg CD, DeMets DL - Fundamentals of clinical trials. London: John Wright; 1981.

7. Shapiro SH, Louis TA - Clinical trials: issues and answers. New York: Marcel Dekker; 1983.

8. Pocock SJ - Clinical trials: a practical approach. Toronto: John Wiley \& Sons; 1983.

9. Plaisier PW, Berger MY, van der Hul RL, Nijs HG, den Toom R, Terpstra OT, Bruining HA Expected difficulties in randomizing patients in a surgical trial: a prospective study comparing extracorporeal shock wave lithotripsy with open cholecystectomy. World J Surg. 1994;18(5):769-72.

10.Schulz KF, Grimes DA - Unequal group sizes in randomised trials: guarding against guessing. Lancet. 2002;16;359(9310):966-70.

11.Sackett DL, Hoey J - Why randomized controlled trials fail but needn't: a new series is launched. Stud Health Technol Inform. 2000;77:29-32.

12.Jadad A - Randomised controlled trials. London: BMJ Books; 1998.

13.MMath HG, McEntegart J, Byrom B, Ghani S \& Shepherd S - Minimization in crossover trials with non-prognostic strata: theory and practical application. J Clin Pharm \& Therap. 2001;26(2): 121. 